\section{Migración por estilo de vida: ¿Creando comunidades diversas y cohesionadas? El caso de Los Riscos, Pucón, Chile*}

Lifestyle migration: Creating diverse and cohesive communities? The case of Los Riscos, Pucón, Chile.

\section{LUIS VeRgara}

Consuelo SÁNCHEZ ${ }^{* * *}$

Hugo Marcelo Zunino**

* Este artículo fue financiado por la Corporación Nacional de Investigación Científica y Tecnológica (CONICYT), a través del proyecto FONDECYT 1150944 "Espacios discursivos alternos en la Araucanía andino-lacustre: imaginando y creando la identidad del lugar".

** Doctorando en Arquitectura y Estudios Urbanos. Pontificia Universidad Católica de Chile. Ilvergara@uc.cl

*** Dirección de Cooperación Internacional. Núcleo de Ciencias Sociales y Humanidades. Universidad de La Frontera, Temuco, Chile.consuelo.sanchez@ufrontera.cl

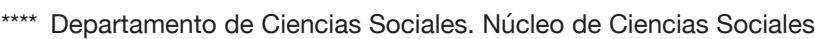
y Humanidades. Universidad de la Frontera, Temuco, Chile. Hugo. zunino@ufrontera.cl

\section{Resumen}

Basados en el estudio de un sector suburbano de Pucón, este trabajo busca analizar cómo la migración por estilo de vida ha impactado en la segregación sociocultural de una comunidad históricamente homogénea en términos socioeconómicos. A través de entrevistas y observación participante, los resultados muestran que la migración ha inducido la gentrificación del área y también ha generado nuevas formas de distinción social. Sin embargo, los resultados muestran que la segregación sociocultural no es consecuencia per se de la diversidad socioeconómica del sector, sino más bien de los distintos reclamos de autenticidad y estilos de vida que conviven en la zona. Sobre esta base se argumenta que la segregación no corresponde a un fenómeno estructural, sino que está anclada en las relaciones entre sujetos $y$, por consiguiente, abierta a transformaciones, especialmente cuando se trata de comunidades suburbanas amenazadas que valoran las amenidades naturales y se oponen a la mercantilización de la naturaleza que las circunda.

Palabras clave: Segregación, migración por estilo de vida, comunidad, gentrificación rural, distinción social.

\begin{abstract}
Based on the study of a suburban sector of Pucón, this work seeks to analyze how lifestyle migration has impacted on the sociocultural segregation of a historically homogeneous community in socioeconomic terms. Through interviews and participant observation, the
\end{abstract}


results show that migration has induced the gentrification of the area and also new forms of social distinction. However, the results also show that the sociocultural segregation is not a consequence per se of the socioeconomic diversity of the area, but rather of the different claims of authenticity and lifestyles that coexist in it. On this basis, it is argued that segregation is not a structural phenomenon but is rather anchored in the relationships between subjects and, therefore, open to transformations, especially when dealing with threatened suburban communities that value natural amenities and oppose the commodification of nature.

Key words: Segregation, lifestyle migration, community, rural gentrification, social distinction.

\section{Introducción}

En Latinoamérica los estudios sobre segregación han tenido recientemente un nuevo impulso debido a la gentrificación y el incremento de la diversidad socioeconómica que ha derivado de este fenómeno (Urrutia-Mosquera et al. 2017; Rasse 2015; Ruiz-Tagle 2016; Sabatini et al. 2001; Saraví 2008; Vignoli y Rowe 2017; LópezMorales 2013; Sabatini et al. 2009; InzulzaContardo 2012; Janoschka et al. 2014). Dichos estudios han puesto su foco sobre la ciudad y sus áreas consolidadas, sin embargo, han ignorado la expresión de este fenómeno en zonas suburbanas y rurales. Avanzando en un tratamiento más equilibrado del fenómeno, este artículo sostiene que en muchos lugares dotados de amenidades paisajísticas, la migración por estilo de vida ha transformado radicalmente las condiciones sociales, culturales y económicas del habitar (Benson y O'Reilly 2009; Nates
2008). Esto ocurre porque en un contexto de creciente urbanización, ha habido un grupo de individuos típicamente urbanos que, agotados de esa condición, han decidido mudarse hacia zonas suburbanas y rurales en búsqueda de un nuevo estilo de vida. A pesar de que no se trata de una migración masiva, está teniendo fuertes repercusiones sobre la diversidad de los lugares receptores, especialmente porque el movimiento involucra a sujetos dotados de un alto capital económico y cultural, que se suman a comunidades tradicionales relativamente homogéneas desde el punto de vista socioeconómico y cultural (Benson y O'Reilly 2009).

La migración por estilo de vida hacia zonas rurales ha despertado el interés de académicos de distintas partes del continente por conocer sus impactos. Los estudios muestran que este fenómeno incrementa el precio del suelo e incentiva la especulación inmobiliaria, creando problemas de acceso a vivienda (González et al., 2009; Zunino et al. 2011; Marchant y Rojas 2015; Sánchez 2017; Kordel y Pohle 2018), desata disputas de ciudadanía especialmente en aquellos entornos donde esta migración involucra personas extranjeras sin derechos legales sobre el lugar (Janoschka 2009; Matossian et al. 2014); impulsa la transformación de las identidades de los migrantes ya sea para integrarse al lugar o reafirmar posiciones de privilegio (Hayes 2015; Kordel y Pohle 2018; Zunino y Huiliñir 2017), así como induce también problemas de desarrollo local y comunitario (Matarrita-Cascante y Stocks 2013; Sánchez 2017). Estos resultados más pesimistas han sido matizados recientemente con trabajos que indican que este tipo de migración revitaliza culturalmente los lugares de acogida e incrementa la diversidad social en ellos (Bennet 
2010; Bell y Jayne 2010; Marchant 2017; Otero et al. 2017).

Sin embargo, aún existe desconocimiento en relación a la manera en que se desarrollan las relaciones sociales entre migrantes y habitantes locales en la vida cotidiana, es decir, hay una brecha de conocimiento respecto a cómo se manifiesta la segregación sociocultural en estos lugares. Si bien estudios recientes en lugares receptores de migración por estilo de vida examinan la "construcción del otro" (Matarrita et al. 2017; Trimano 2017), estos resultados no han sido interpretados en el contexto de las discusiones actuales sobre segregación socioresidencial. Por lo anterior, este trabajo busca analizar la segregación sociocultural de una comunidad suburbana receptora de migrantes por estilo de vida en el sur de Chile. A diferencia de la mayoría de los trabajos realizados hasta ahora, el enfoque aquí está puesto sobre las personas que históricamente han habitado en el lugar, ya que su posición resulta clave para entender el impacto de este tipo de migración sobre la segregación de las comunidades, por cuanto ellos han sido testigos de los cambios producidos por el arribo de nuevos habitantes.

Un aspecto central para conectar la discusión sobre migración por estilo de vida y segregación es la diversidad social. Así como hoy las ciudades avanzan en diversidad, la migración por estilo de vida impulsa heterogeneidad en sectores rurales y suburbanos (Bell y Jayne 2010; Benson y O’Reilly 2009; Florida 2005; Méndez 2014; Stockdale 2010). Ambos fenómenos son paralelos y su estudio puede ayudar a entender la manera en que opera la segregación en entornos de creciente diversidad, aportando con ello no solo al conocimiento de un lugar específico, sino que también en la discusión general sobre segregación.

El artículo se estructura en cinco secciones. En la primera, se conecta la discusión sobre segregación y migración por estilo de vida. Luego se describe el caso de estudio, ubicado en un área suburbana cercana a la comuna de Pucón, localidad que se ha posicionado como una de las principales receptoras de migración por estilo de vida de la norpatagonia chilenoargentina. Después se describe la estrategia metodológica desplegada. A continuación, se presentan los resultados organizados en tres subsecciones mostrando; primero, la gentrificación rural y la percepción que los locales tienen sobre los migrantes; segundo, la manera en que los locales se relacionan con los migrantes por estilo de vida y cómo ello marca distinciones sociales; y tercero, el origen de aquellas distinciones. Se finaliza reflexionando en torno a la necesidad de situar la mirada en la segregación sociocultural en lugares suburbanos y rurales sujetos a procesos de creciente diversidad social.

\section{Segregación y migración por estilos de vida: ¿Comunidades diversas y cohesionadas?: entre la aproximación estructural y la microsocial.}

Dentro de la literatura especializada la segregación, o la separación existente entre grupos sociales, suele ser definida como un fenómeno espacial (White 1983; Massey y Denton 1988; Monkkonen 2012; Sabatini et al. 2001). Esta forma de entender la segregación hace hincapié en la manera en que las familias se distribuyen geográficamente al interior de un territorio. Por ejemplo, en un influyente trabajo, 
Massey y Denton (1988) la han definido como "el grado en que uno o más grupos viven separados entre sí en diferentes partes del entorno" (p. 282). Por cierto, la segregación tiene una dimensión espacial, pero como lo han mostrado trabajos recientes, la separación entre grupos sociales es un fenómeno que también involucra una dimensión social o cultural (Sabatini et al. 2001; Saraví 2008; Vergara-Erices y Garín 2016). Esta dimensión sitúa su foco sobre la sociabilidad y el reconocimiento que existe entre diferentes sujetos (Bayón y Saraví 2013; Rasse 2015), lo que hace referencia directa también a la cohesión social y los procesos que subyacen en la construcción de comunidades (Rasse 2015; Cassiers y Kesteloot 2012). Esta última es la manera en que este artículo entiende y analiza a la segregación socio-residencial: como un fenómeno asociado a la manera en que se manifiestan las relaciones sociales entre las personas y la forma en que ellas se reconocen mutuamente, procesos que influyen sobre la construcción de comunidades sociales.

La dimensión espacial y la cultural de la segregación pueden operar de forma dialéctica (Sabatini et al. 2001; Saraví 2008; VergaraErices y Garín 2016). Un territorio altamente homogéneo, es decir, segregado espacialmente, puede no segregarse desde el punto de vista cultural. Esto ocurre, por ejemplo, en lugares socialmente homogéneos como barrios cerrados y/o guetos, en los que suelen haber comunidades bien cohesionadas internamente (Manzi y Smith-Bowers 2005; Villarreal y Silva 2006). La dialéctica de la segregación también puede ocurrir en territorios heterogéneos, es decir, no segregados espacialmente, pero que pueden estar cruzados por conflictos y distinciones de distinto tipo que impiden la construcción de una comunidad. La forma contradictoria en la que se relaciona el espacio físico y social, da cuenta de la complejidad en la que se manifiesta la segregación.

Existe bastante respaldo académico para sostener que la migración por estilo de vida incrementa la heterogeneidad socioeconómica de los lugares (Bell y Jayne 2010; Benson y O'Reilly 2009; Florida 2005; Phillips 2005; Méndez 2014; Stockdale 2010; Nogué 1988). Este tipo de migración involucra el movimiento de individuos dotados de un capital económico y cultural alto, típico de clases media alta o clase alta, hacia lugares relativamente homogéneos y tradicionales desde el punto de vista económico y cultural (Bell y Jayne 2010; Benson y O'Reilly 2009; Florida 2005; Méndez 2014; Stockdale 2010). Por esta razón, el arribo de migrantes no solo incrementa la heterogeneidad socioeconómica de los lugares receptores, sino que también establece las bases para innovar culturalmente y transformar la economía de ciudades pequeñas o zonas rurales (Bell y Jayne 2010; Marchant 2017). Más allá de estos aspectos aparentemente positivos, este movimiento también está acompañado de especulación inmobiliaria e incremento en el precio del suelo (Hidalgo y Zunino 2011; Zunino et al. 2011; Marchant y Rojas 2015), lo que termina impulsando el desplazamiento de la población local (Stockdale 2010; Phillips 2005). Los problemas de acceso a la vivienda y el perfil de clase media-alta que conduce la migración por estilo de vida, han motivado el desarrollo y empleo del concepto de gentrificación rural para describir las transformaciones que acontecen en lugares que reciben este tipo de migración (Phillips 2005; Stockdale 2010; López-Morales 2018). Sin embargo, esto también ha sido objeto de crítica, primero, por ser una adaptación de un fenómeno típicamente urbano, lo que 
representaría la colonización epistémica de lo rural (Bernt 2018), y segundo, porque no siempre produce desplazamiento de antiguos residentes de bajos ingresos, aspecto central en las definiciones de gentrificación (Halfacree 2018).

Pero, ¿qué ocurre con la segregación de los territorios receptores de migración por estilo de vida? Si entendiéramos la segregación solo como un fenómeno espacial, la respuesta sería que la migración acerca espacialmente a grupos diferentes, lo cual puede considerarse como positivo. Pero si entendiéramos la segregación como un fenómeno sociocultural y dialéctico, la respuesta no necesariamente sería tan clara. Para el caso latinoamericano, Trimano (2017) ha sostenido recientemente que la migración por estilo de vida impulsa conflictos locales. Los habitantes históricos del lugar generalmente se sienten desplazados por los migrantes, quienes intentan imponerles una forma de vida diferente. En este proceso, los habitantes locales se diferencian de los migrantes, a quienes reconocen como afuerinos, quienes no tienen un lazo histórico con el territorio en el que habitan. En Chile, Matarrita et al. (2017) han investigado la percepción de otredad entre los migrantes y los habitantes locales y cómo esta afecta el desarrollo local de las comunidades. Basándose en el estudio de Malalcahuello, un destino de migración emergente (Marchant y Rojas 2015), los autores describen como las diferencias económicas y culturales existentes entre los migrantes y habitantes locales establecen una distinción per se entre el "ellos" y el "nosotros" y esas distinciones se intensifican por el escaso contacto que hay entre ambos grupos al interior de las localidades.

Con base en los estudios aquí mencionados, se puede establecer que la relación entre migrantes y habitantes locales ocurre en esferas de sociabilidad diferenciadas. Mientras los migrantes construyen comunidad relacionándose mayoritariamente entre ellos, los habitantes locales hacen lo mismo, pero aislados de los migrantes. Existe por tanto separación entre ambos grupos y consecuentemente segregación sociocultural. Aparentemente la proximidad espacial entre los grupos en cuestión-que es característica de las pequeñas localidades receptoras de este tipo de migración- no es suficiente para hacer que se produzcan procesos de integración entre migrantes y locales. Como apunta Matarrita et al. (2017) la raíz de aquella diferenciación está en las diferencias socioeconómicas que existen entre los grupos. Es decir, para los autores, se establece una conexión entre segregación y condición socioeconómica que hace difícil la construcción de comunidades en lugares caracterizados por la diversidad de ingresos.

En los estudios de segregación, ha habido autores que han criticado la relación mecánica entre diversidad socioeconómica y construcción de comunidades. Esta aproximación, que ha sido llamada "tesis del espejo" (Sabatini et al. 2001) por entender que las desigualdades sociales se reflejan en el espacio y en las relaciones entre los sujetos, ha sido matizada por una aproximación que pone su mirada sobre las personas como sujetos con capacidad de agencia. Desde esta última aproximación, más microsocial, la construcción de comunidades en lugares caracterizados por la diversidad sería perfectamente posible, ya que a pesar de que los estilos de vida y valores sociales están influenciados por la posición social (Inglehart 1990; Bourdieu 2002), la cohesión no estaría completamente estructurada por esta. Por el contrario, la comunidad estaría posibilitada por 
las acciones que las personas emprenden en un momento y entorno específico.

Como Benson (2013) ha mostrado, un aspecto clave para entender la formación de comunidades en lugares receptores de este tipo de migración, y desde una perspectiva más microsocial, son los reclamos de autenticidad. Para la autora, los lugares rurales idílicos tienen la capacidad de satisfacer el deseo de los migrantes por sentirse auténticos, reconectándolos con la naturaleza y la esencia de la vida. No obstante, emergen obstáculos para la creación de comunidades dado que los migrantes se encuentran con intereses locales diferentes, que en ocasiones incluso apoyan el avance de artefactos modernos, como por ejemplo centros comerciales (ver por ejemplo caso de Puerto Varas en Chile en Vergara et al. 2013). Estas situaciones crean conflictos y formas de distinción social entre locales y migrantes que fortalecen la separación identitaria y obstaculizan la construcción de comunidades cohesionadas.

No obstante, desde una perspectiva microsocial, la construcción de comunidades diversas y cohesionadas no sería necesariamente imposible. Para ello serían claves las actitudes de integración hacia los locales que manifiesten los migrantes. A pesar de que una buena parte de los migrantes por estilo de vida tienden a poseer un discurso individualista (Korpela 2014), hay también quienes apuestan por valores más comunitarios (Zunino y Huiliñir 2017). Estas diferencias entre migrantes por estilo de vida, producen diferentes actitudes de integración con la población local. En este contexto, la diversidad socioeconómica impulsada por la migración por estilo de vida no sería un obstáculo per se para la construcción de comunidades, más bien, este proceso operaría a nivel microsocial y a nivel de vida cotidiana, estando permeado por la actitud de integración que manifiestan los migrantes con la comunidad local.

En definitiva, más allá de las diferentes aproximaciones disponibles y formas de interpretación de la relación existente entre migración y segregación, los resultados parecen ser igualmente pesimistas: se ha instalado la idea de la migración por estilo de vida, aunque aporta en diversidad, no puede contribuir a crear comunidades cohesionadas. Entre migrantes y locales emergen formas de distinción social que potencialmente pueden derivar en conflictos sociales. Estos conflictos establecen una relación simbiótica con las formas de distinción, en el sentido de que ambos se refuerzan mutuamente. Sin embargo, más allá de este diagnóstico común, no hay acuerdo si efectivamente esos conflictos sociales que refuerzan la segregación emergen por aspectos estructurales, es decir, naturalmente por la existencia de diversidad socioeconómica, o más bien están impulsados por la propia dinámica microsocial que adquieren las relaciones en estos lugares.

\section{Caso de estudio: Los Riscos, Pucón, Chile}

Los Riscos está enclavado en la Araucanía Andina chilena, a $9 \mathrm{~km}$ de Pucón y a $17 \mathrm{~km}$ de Villarrica. La localidad corresponde a un sector suburbano rodeado de una amplia gama de recursos naturales que incluyen bosques nativos, humedales, el lago Villarrica y el volcán del mismo nombre.

Los Riscos, se caracteriza hoy por ser un sector heterogéneo desde el punto de vista 
socioeconómico y de los estilos de vida que ocurren en él. En términos generales, se pueden observar dos grupos. Por un lado, están los habitantes locales, grupo constituido por varias generaciones de familias y con tradiciones arraigadas. En su mayoría son propietarios históricos de los terrenos, los que albergan a familias extensas compuestas por adultos mayores que conviven junto a hijos y nietos. Su forma de habitar está regida por un estilo rural campesino, que pone énfasis en las tradiciones y actividades económicas asociadas a trabajos domésticos y la explotación de la tierra. Por otro lado, están los migrantes por estilo de vida. Estas son familias pequeñas, relativamente jóvenes, con alto capital económico y cultural que han migrado desde grandes ciudades buscando un estilo de vida alternativo, el contacto con la naturaleza y la libertad.

La diversidad socioeconómica de Los Riscos se observa en los tipos de vivienda que hay en el sector ${ }^{1}$. Están las viviendas unifamiliares, de diversas fachadas y materiales constructivos, que albergan tanto a migrantes por estilo de vida como a habitantes locales. También hay condominios, que aportan con la mayor cantidad de viviendas en el sector, algunos constituidos por viviendas unifamiliares de gran tamaño y de diseño tradicional, mientras que otros optan por viviendas de diseño autosustentable.

La fachada de la casa se ha convertido en uno de los principales indicadores subjetivo de las condiciones socioeconómicas de las familias, especialmente en una sociedad chilena donde el consumo está masificado (Ariztía, 2009; Ruiz-Tagle, 2016). Desafortunadamente los datos del CENSO 2002 son muy antiguos para mostrar el cambio social de Los Riscos, el cual ha sido más reciente. Asimismo, el CENSO 2017 solo entrega información sobre el nivel educacional como variable de aproximación a la condición socioeconómica de la población a nivel de manzana o distrito censal. Sin embargo, la escolaridad tampoco es un buen indicador de la condición de ingresos en una sociedad en la que la educación superior alcanza amplia cobertura (Espinoza y Barozet, 2009).
Asimismo, es posible encontrar parcelas de agrado con residentes permanentes o utilizadas como segunda vivienda durante la época estival. Tanto las parcelas como las viviendas en condominios son habitadas mayoritariamente por migrantes. Según registros de "Portal Inmobiliario", sus valores de venta alcanzan hasta los 278.000 .000 pesos chilenos (457.970 USD aproximadamente). No hay un lugar específico dentro de Los Riscos que concentre cada tipo de vivienda, por el contrario, estas se encuentran distribuidas aleatoriamente en el sector, creando un panorama de mixtura socioespacial que tiene a habitantes locales tradicionales habitando en proximidad con nuevos residentes. Cabe destacar que la heterogeneidad de Los Riscos es un fenómeno que no tiene más de 15 años, siendo la migración el fenómeno que ha transformado esta comunidad que históricamente había estado caracterizada por un tradicional estilo de vida campesino.

Los migrantes por estilo de vida, (nacionales y extranjeros) se han insertado a nivel cultural, social y económico dentro de la localidad. Su integración se ha dado especialmente a través de un Colegio que ofrece pedagogía Warldof ubicado en el sector y la participación activa de algunos de ellos en la Junta de Vecinos. El colegio está abierto tanto para las personas del sector como para habitantes de Pucón y alrededores que buscan un sistema educativo alternativo para sus hijos. Además, este espacio cumple el papel de eje cultural dentro del sector gracias a las actividades que imparte de forma

Portal Inmobiliario es una web que alberga viviendas a la venta en diferentes lugares de Chile. El valor promedio de venta fue calculado a partir de las casas y departamentos nuevos que estaban siendo ofrecidos en la zona de Los Riscos en Pucón con fecha Abril de 2018. 
abierta a toda la comunidad, como ferias de trueque y talleres basados en la sustentabilidad. La relación entre migrantes y locales se ha dado también en el plano laboral. Los primeros han creado empleos vinculados a servicios domésticos en los que participan los residentes históricos del lugar. Estos espacios han permitido que los locales y migrantes por estilo de vida se conozcan y relacionen al interior de los Riscos.

\section{Estrategia metodológica}

En Latinoamérica la migración por estilo de vida, como fenómeno sociocultural y espacial, ha sido estudiada mayoritariamente por medio de indicadores cuantitativos (precio de suelo, permisos de construcción, permisos comerciales) (González 2011; Marchant y Rojas 2015; Hidalgo y Zunino 2011), entrevistas a personas asociadas a la planificación territorial

Figura $n^{\circ}$ 1. Esquema de localización del área de Estudio.

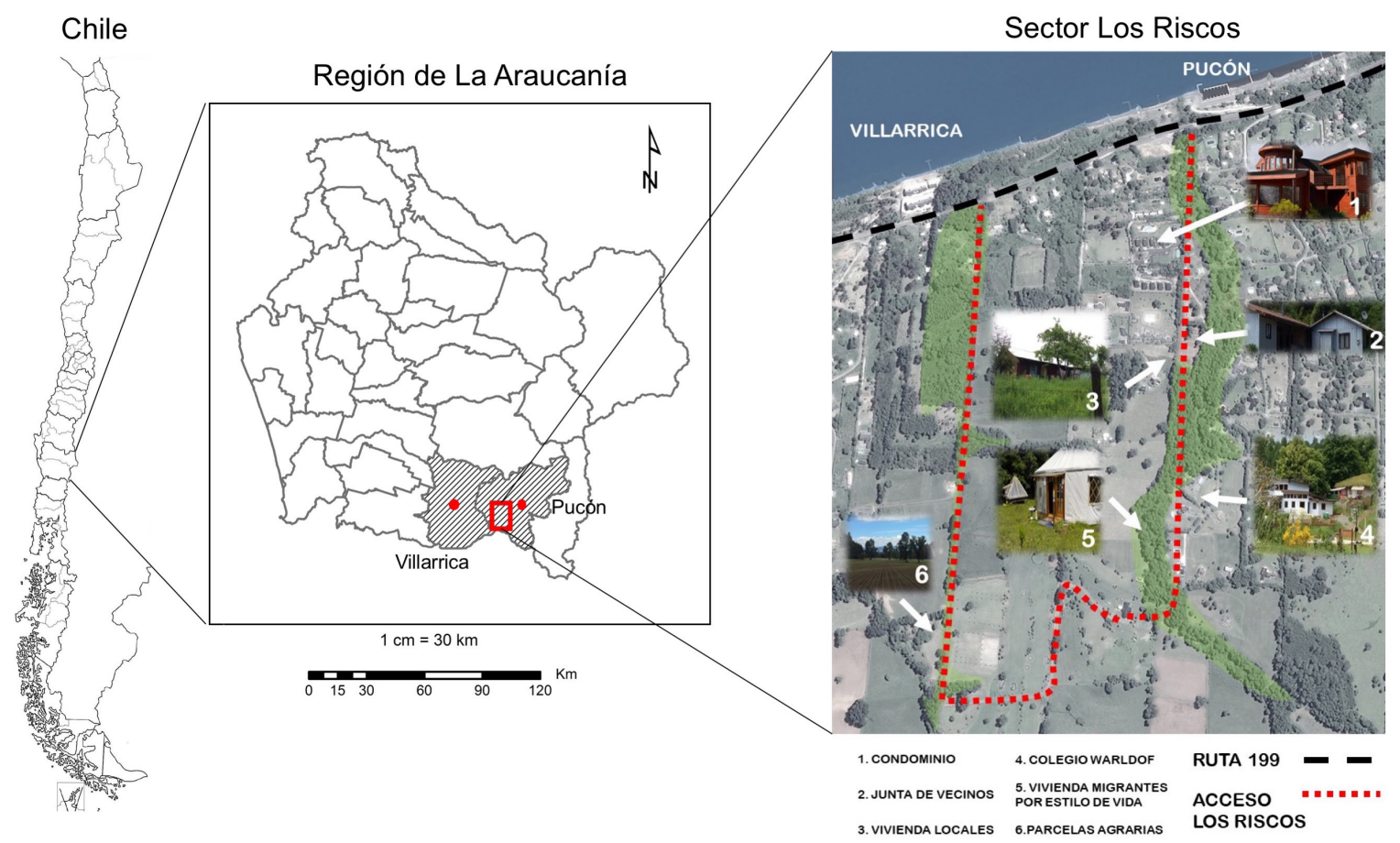

Fuente: elaboración propia. 
local (González 2011; Sánchez 2017; Vergara et al. 2013) o bien a través del estudio de los propios migrantes (González et al. 2009; Hayes 2015; Kordel y Pohle 2018; Marchant 2017; Matarrita et al. 2017; Méndez 2014; Otero et al. 2017; Zunino y Hidalgo 2010). Esto significa que ha habido poca atención sobre los habitantes locales y en la forma en que ellos leen este proceso. Este vacío nos parece particularmente importante, ya que ellos son los actores en mejor posición para evaluar las transformaciones derivadas de la migración, entre ellas, las vinculadas a la segregación. Es por lo anterior que en esta investigación se desarrolló una aproximación desde el punto de vista de los habitantes locales, entendiéndolos como claves para entender los efectos de la migración sobre la segregación. En específico, se realizó un estudio exploratorio cualitativo que empleó observación participante, el método conversacional y una entrevista en profundidad como técnicas para recolectar datos. Esta estrategia de triangulación metodológica permitió hacer más confiables los resultados del trabajo (Okuda y Gómez-Restrepo 2005).

Los tres instrumentos fueron aplicados a 12 habitantes del sector Los Riscos durante dos trabajos de campo desarrollados en diciembre de 2017. La muestra fue definida a partir de saturación teórica, es decir, cuando las respuestas entregadas por los entrevistados y evidencia levantada, comenzó a ser repetitiva. El acceso a los entrevistados fue a través de la técnica bola de nieve, contando con el apoyo inicial de algunos dirigentes del sector que, posterior a varias conversaciones, invitaron a los investigadores a participar en la fiesta navideña del sector y a colaborar en una actividad con los niños de la localidad. En el primer trabajo de campo se sostuvieron conversaciones con lugareños, examinando la percepción que ellos tenían respecto al cambio poblacional del lugar, el origen social y territorial de los nuevos vecinos, los cambios territoriales y de propiedad en la zona, los espacios de encuentro comunitarios y el aporte de los migrantes en términos culturales y laborales. Una vez realizado el primer acercamiento a la comunidad, se procedió a analizar los resultados preliminares a través de una codificación abierta la que dio como resultado la incorporación de nuevos tópicos de examen. Entre ellos se incluyeron la relación con los migrantes, los conflictos locales, los estilos de vida del lugar y cómo había cambiado la cultura social del sector a partir de la llegada de nuevos vecinos. Con esta base se realizó un segundo terreno de trabajo que involucró entrevistas, conversaciones y observaciones con una buena parte de los habitantes locales. Ese trabajo de terreno se desarrolló en paralelo a la actividad navideña con los niños, capturando la opinión y sensibilidad respecto a los efectos de la migración sobre la segregación social del sector. Cabe consignar que a dicha actividad también estaban invitados los migrantes, sin embargo, ninguno de ellos concurrió. Esto generó un ambiente de libertad y confianza en los habitantes locales, pudiendo estos conversar abiertamente sobre sus inquietudes, reflexiones y críticas respecto a los migrantes, permitiendo así mantener cierto "compromiso conversacional" (Goffman 1974).

La muestra de las entrevistas quedó compuesta por personas de diferentes grupos etarios, niveles educacionales y dependencias laborales. Dentro de las características de la muestra destaca particularmente que el $75 \%$ de las personas entrevistadas ha vivido por más de 10 años en el sector, por lo que han sido testigos claves de los cambios ocurridos en el sector a partir del arribo de nuevos residentes. 
Tabla n¹. Caracterización de la muestra.

\begin{tabular}{|c|c|c|c|}
\hline \multicolumn{2}{|c|}{ Variable } & $\mathbf{N}^{\circ}$ & $\%$ \\
\hline \multirow{5}{*}{$\begin{array}{l}\text { Total } \\
\text { integrantes } \\
\text { grupo familiar }\end{array}$} & De 1 a 2 & 3 & $25 \%$ \\
\hline & De 3 a 4 & 3 & $25 \%$ \\
\hline & De 5 a 6 & 1 & $8 \%$ \\
\hline & De 6 a 7 & 3 & $25 \%$ \\
\hline & Mas & 2 & $17 \%$ \\
\hline \multirow{5}{*}{ Edad } & $18-26$ & 3 & $25 \%$ \\
\hline & $26-40$ & 3 & $25 \%$ \\
\hline & $40-50$ & 2 & $17 \%$ \\
\hline & $50-65$ & 1 & $8 \%$ \\
\hline & mas de 65 & 3 & $25 \%$ \\
\hline \multirow{8}{*}{$\begin{array}{l}\text { Nivel de } \\
\text { escolaridad }\end{array}$} & Básica incompleta & 4 & $34 \%$ \\
\hline & Básica completa & 1 & $8 \%$ \\
\hline & Media incompleta & 2 & $17 \%$ \\
\hline & Media completa & 3 & $25 \%$ \\
\hline & Técnica superior & - & - \\
\hline & $\begin{array}{l}\text { Universitaria } \\
\text { incompleta }\end{array}$ & 1 & $8 \%$ \\
\hline & $\begin{array}{l}\text { Universitaria } \\
\text { completa }\end{array}$ & 1 & $8 \%$ \\
\hline & Postgrado & - & - \\
\hline \multirow{6}{*}{$\begin{array}{l}\text { Dependencia } \\
\text { laboral }\end{array}$} & Dependiente & 2 & $17 \%$ \\
\hline & Independiente & 2 & $17 \%$ \\
\hline & Jubilado & 2 & $17 \%$ \\
\hline & Buscando trabajo & - & - \\
\hline & Dueña de casa & 5 & $41 \%$ \\
\hline & Otra & 1 & $8 \%$ \\
\hline \multirow{5}{*}{$\begin{array}{l}\text { Cantidad de } \\
\text { años en el } \\
\text { sector }\end{array}$} & $0-5$ & 3 & $25 \%$ \\
\hline & 5 a 10 & - & - \\
\hline & 10 a 15 & 1 & $8 \%$ \\
\hline & 15 a 20 & - & - \\
\hline & mas de 20 & 8 & $67 \%$ \\
\hline
\end{tabular}

Fuente: elaboración propia.
Se registró el audio de todas las conversaciones y los nombres de los entrevistados han sido modificados para resguardar su identidad. Todas las entrevistas y conversaciones fueron analizadas con la ayuda del software Atlas.ti. desarrollando a partir de él una codificación abierta que permitió ordenar las diferentes respuestas de los participantes sobre cada uno de los tópicos examinados.

\section{La transformación del lugar y el arribo de nuevos vecinos: la gentrificación de los migrantes por estilo de vida.}

Los habitantes locales perciben que los Riscos ha cambiado de forma notable en la última década, física y socialmente. Uno de los primeros cambios que plantean se vincula a la expulsión de los antiguos habitantes del sector. El incremento en el precio de los terrenos y el interés de inversores y particulares por comprar la tierra de Los Riscos, ha incentivado a muchos propietarios de este lugar a vender sus terrenos. A eso se ha sumado el deseo de migrar hacia la ciudad que han mostrado varios de los habitantes que crecieron en la zona y que hoy son adultos jóvenes. Una buena parte de estas personas han decidido no continuar viviendo en el sector, motivados también por retornos económicos que pueden obtener a partir de la venta de terrenos generalmente heredados de sus padres. Así describe una entrevistada los cambios ocurridos a partir de la venta de terrenos en el sector:

Es que ahora esto es otro lugar, una zona turística total. Aquí estamos quedando nosotros y un hermano. Acá arriba condominios, por todos lados. Estamos rodeados de condominios (...) Todos han vendido, pequeñas parcelas y se instalan por todas partes (...) Aquí hay un caballero que tiene mucho dinero, él compró 100 hectáreas en 
Correntoso -la entrada del sector los Riscos- y las loteó. Lotea y vende el sitio a gente de dinero y [ellos] vienen a hacer sus casas y viven acá, o sea, ya no es gente que viene a veranear, sino que ahora viven acá (Verónica).

No es extraño que Los Riscos siendo un sector suburbano de una ciudad turística como Pucón, esté experimentando un proceso de incremento en el precio del suelo. Dicho proceso ya ha sido evidenciado en otras pequeñas ciudades turísticas dotadas de amenidades paisajísticas por todo el globo y, como fue examinado, ha sido conceptualizado bajo la idea de gentrificación rural. Este fenómeno genera una serie de inconvenientes en los habitantes que históricamente han residido en estos lugares. Una habitante local se expresa en los siguientes términos respecto a la dinámica mercantil que se ha apropiado del lugar:

Investigador: ¿Alguna vez le han ofrecido dinero para comprar su casa o su terreno?

\begin{abstract}
Si, todo el tiempo hay gente interesada. Pero lo que pasa es que yo creo que ya no quedan sitios en Los Riscos donde comprar. Si tú quieres buscar, no vas a encontrar un terreno. Ahora no hay nada. $Y$ han subido mucho los precios. 1/2 hectárea sale 50 millones de pesos (Amalia).
\end{abstract}

Los datos de precios de viviendas y terrenos que posee el Servicio de Impuestos Internos corroboran lo planteado por la entrevistada. Según este organismo público, que tasa el valor de las propiedades, la mayor parte de los terrenos de Los Riscos tiene una superficie de $1 / 2$ hectárea $(5.000 \mathrm{~m} 2)$. Para el primer semestre de 2019 el valor comercial de dicha superficie suele rondar los \$52.074.579 de pesos chilenos para terrenos sin construcción asociada, mientras que varía entre $\$ 118.662 .549$ y $\$ 161.828 .827$ millones en terrenos con viviendas construidas, precios que dependen esencialmente de la cercanía al lago Villarrica, la vista hacia el volcán del mismo nombre y el acceso que los terrenos tengan a caminos que conectan el área con las ciudades circundantes ${ }^{3}$.

Hoy la oferta de terrenos es acotada. La mayoría del suelo ya ha sido traspasado a nuevos residentes y la migración concomitante de habitantes locales por venta de terreno a perdido intensidad. Así, hoy es particularmente difícil encontrar familias que hayan habitado allí por más de 10 años. Se han mantenido en el lugar las familias con mayor arraigo, quienes resisten el impulso por vender para preservar el vínculo afectivo con el área.

La dinámica económica de la zona ha sido el mecanismo central en la gentrificación del área. Tal como ocurre con la gentrificación urbana que opera en lugares previamente deteriorados, Los Riscos y la actividad agrícola que sustentaba la dinámica económica local experimentaron un proceso de deterioro económico importante durante los años noventa del siglo pasado. Esto, sumado a la concentración de servicios en las áreas urbanas circundantes (Pucón y Villarrica), significó que el área perdiera su atractivo como un lugar para residir, especialmente entre los adultos jóvenes y descendientes herederos de los antiguos habitantes locales. Una vez deteriorada el área, arribó con fuerza el turismo y la inversión inmobiliaria a la zona. Este proceso ocurrió especialmente durante la década pasada y permitió a inversores individuales y empresas inmobiliarias comprar terrenos a precios más bajos. Hoy, dado que la zona ha recuperado su atractivo, los precios se han elevado considerablemente.

En Chile la tasación fiscal realizada por el Servicio de Impuestos Internos representa en promedio el 54\% del valor comercial de los lugares (Ruiz-Tagle et al. 2018). Sobre esa base fue calculado el valor aquí expresado. 
La percepción de los entrevistados es concomitante con lo que muestran las estadísticas, respecto a que en las últimas décadas los Riscos ha incrementado de forma considerable sus habitantes. Consultadas por el cambio poblacional de la zona, los habitantes locales reflexionan:

La población ha crecido, si hablamos de porcentaje, un $70 \%$, porque todavía quedan varias familias antiguas, pero son 2 o tres, de las antiguas (Isabel).

Esto fue así como un boom, digamos unos ocho años atrás, paso de ser campo, una zona rural, a ser ya semiurbano (...) se ha poblado mucho, mucha población. Lo que eran campos particulares... la gente ha estado vendiendo (...) y todo se está poblando, por todos los alrededores. Solo estamos quedando pocas personas que tienen un poco más de campo porque lo demás está todo poblado (Verónica)

La expulsión de la población tradicional $y$ el arribo de nuevos habitantes han sido procesos complementarios que no solo han transformado físicamente el sector a través de las parcelaciones, sino que también han cambiado el perfil social de sus habitantes. En el pasado los Riscos era un típico lugar rural chileno: conformado por personas dedicadas mayoritariamente a la agricultura y con bajo capital cultural y económico. Hoy, los nuevos habitantes tienen un perfil marcadamente diferente. Según expresan los entrevistados, la migración ha permitido incrementar la diversidad económica y cultural del lugar y aquello es un aspecto que no es interpretado negativamente, por el contrario, es bien apreciado por los locales. Así lo expresa un dirigente social del área:

Aquí hay distintas clases sociales dentro de un mismo sector. Tienes clase baja, clase media, clase alta y muy alta. Cuatro grupos sociales diría yo (...) es un lugar diverso. Eso es bueno, porque hay realidades que yo no conocía (Javier).
La expulsión de los habitantes históricos, sumada al recambio de estos por residentes con un perfil socioeconómico más alto, consolida el proceso de gentrificación rural en el lugar. Desde este fenómeno es como se entienden los cambios físicos y sociales que han ocurrido en el área durante la última década.

¿Cómo caracterizan los locales a sus nuevos vecinos? Lo hacen a partir de su forma de vida y sus propósitos culturales. Más allá de que sean personas dotadas de mayor acceso a recursos, lo que más llama la atención de los locales es la relación que los migrantes establecen con el medioambiente que los rodea y sus prácticas cotidianas. Varios de los entrevistados hicieron referencia a que los nuevos vecinos cuidaban el medioambiente a través de prácticas de compostaje y reciclaje. También, destacaron que ellos intentaban intervenir lo menos posible sobre el entorno, practicando en algunos casos una agricultura de subsistencia e incluso actividades de recolección que les permitían obtener lo necesario para alimentarse. Asimismo, los caracterizaron como personas más relajadas, que veían el mundo desde otra perspectiva, sin necesidad de acelerar los procesos naturales ni sociales. Todas estas características hacen que los habitantes locales llamen a sus nuevos vecinos como "hippies", sin que este apelativo tenga necesariamente un sentido negativo.

No obstante, un habitante del lugar advierte que no todos los migrantes son iguales. El migrante "pudiente" o "cuico", como también lo llaman en la zona, es aquel que tiene una buena situación económica - probablemente clase alta o media-alta, trabaja en la ciudad, posee estudios universitarios y está poco tiempo en el sector. Mientras que el migrante "hippie-hippie" 
se caracteriza por cuidar del medioambiente, posee una situación económica asociada a clase media y media-baja, como también estar gran parte del día en Los Riscos. Así, la construcción del imaginario que los locales tienen sobre sus nuevos vecinos se mueve entre dos polos: entre aquellos que, aunque diferentes, aún se encuentran insertos dentro del sistema laboral; y aquellos que más bien están al margen del sistema y disfrutan de una vida alejada del ajetreo cotidiano de las urbes.

La gentrificación observada en la zona está motivada por sujetos que, aunque mayoritariamente de clase media, poseen intereses diferentes a los documentados en los estudios urbanos: en este caso se trata de migrantes con valores anti-urbanos $y$ profundamente interesados en el cuidado de la naturaleza y lo rural. No obstante, y a pesar de sus buenas intenciones, parecen estar generando cambios importantes en la geografía social del área, como la expulsión de los residentes locales y el incremento en el precio de suelo. Esta paradoja se explica porque los intereses y decisiones de cambio de residencia de los migrantes están mediados por el funcionamiento del mercado inmobiliario, el cual ha observado en este tipo de individuos una nueva modalidad de capitalizar el suelo rural. Por eso, más allá que este grupo de personas tenga en mente la realización de un proyecto de vida alternativo, sus prácticas cotidianas no están completamente desprendidas de la manera en que opera territorialmente el sistema capitalista, especialmente en aquellos migrantes que desarrollan su cotidianeidad en las ciudades circundantes. La duda que emerge respecto al papel que los migrantes han tenido en la transformación del lugar, lleva la pregunta hacia la relación que los locales establecen con ellos.

\section{Sociabilizando con migrantes: tejiendo la relación con una "comunidad de extraños"}

La relación entre los locales y los nuevos residentes se produce mayoritariamente en dos espacios de encuentro: la Junta de Vecinos y el colegio ubicado en la localidad. El primero es una institución local de larga data a la cual se han integrado los migrantes durante los últimos años. Allí se produce un encuentro con fines más políticos, relación que se ha traducido en el impulso de proyectos conjuntos que involucran a toda la comunidad. Si bien la incorporación de los migrantes a esta instancia ha generado algunos problemas de poder $-a$ los que se hará mención más adelante- esta ha sido valorada por los locales, ya que el mayor capital cultural con el que cuentan los migrantes les ha permitido adjudicarse fondos públicos para el desarrollo de iniciativas en la zona. Así se describe el proceso de integración de los migrantes a algunas instituciones de Los Riscos:

Sí se han tratado de integrar a la junta de vecinos. A la iglesia nada, pero si a la junta de vecinos. Hay algunos que están participando ya en la junta de vecinos como integrantes de la directiva (Verónica).

La iglesia, institución que tiene importancia especialmente entre los habitantes locales mayores, no opera como un espacio de encuentro con migrantes. Según lo que señalan los locales, una buena parte de los migrantes profesan otro tipo de religión, desconocida para ellos, o simplemente no están interesados en ninguna creencia religiosa. Las entrevistas muestran que esta diferencia no se traduce en conflictos.

Además de la Junta de Vecinos, existe en la zona un espacio de encuentro que se ha construido 
bajo los principios de los migrantes y al cual esporádicamente son invitados los locales: el colegio Waldorf Pucón. Esta institución se trasladó hasta la zona en 2012 y desarrolla un proyecto educativo inspirado en la antroposofía y en la pedagogía alternativa inspirada por esta visión. Este espacio se ha constituido como el lugar de encuentro predilecto entre migrantes, no solo de la zona, sino que también de Pucón y sectores suburbanos y rurales cercanos. Como lo indican los entrevistados, la comunidad local es invitada regularmente a participar en actividades que se realizan en el colegio:

\begin{abstract}
Ellos siempre nos hacen invitación cuando tienen actividad en la comunidad del colegio, que es donde se juntan todos los papas, porque sus hijos van a ese colegio. Entonces actividad que hacen, ya sea una feria o trueque que tengan ellos, invitan a la comunidad y los vecinos a que sean partícipes de su actividad (Isabel).
\end{abstract}

Más allá de la participación en talleres, los habitantes locales no tienen mayor presencia en la escuela. Sus hijos -como plantean los entrevistados - no participan de las clases lectivas que se ofrecen en el lugar, ya que los aranceles anuales superan el 1.870.000 (CLP) (3.800 USD) $)^{4}$, valor que excede la capacidad de pago del habitante local. En ese contexto, la escuela parece operar como un reducto migrante que, aunque en armonía con el medioambiente natural por medio de sus principios de enseñanza, excluye a la mayoría de los locales.

Pero la escuela no solo alberga clases lectivas, sino que también ferias y talleres que son ofrecidos a toda la comunidad. Algunos de los entrevistados valoran positivamente esto, sosteniendo que la escuela les ha enseñado una

Información obtenida desde http://www.waldorfpucon.org/. Febrero, 2018. nueva forma de vida sobre la base de principios de vida inspirados por una concepción místicoespiritual del mundo. Así lo plantea una entrevistada:

\begin{abstract}
Nos han enseñado a vivir con eso, con que nosotros podemos reducir en gran cantidad la basura, el tema de ir separando, el tema de reciclar. Ellos nos han enseñado mucho también sobre la autoproducción y también ellos trabajan con el tema del trueque. Ellos hacen intercambio en vez de venderte algo. ¡Oye sabes que tengo tal cosa!, pero ite lo puedo cambiar por esto? Entonces ellos trabajan con ese tipo de cosas, trueque, ya sea de cosas materiales, de ropa, etc. (Verónica).
\end{abstract}

El contacto entre locales y migrantes también se ha manifestado a través del surgimiento de vínculos laborales. Aunque este tipo de contacto es poco común, el arribo de migrantes ha abierto puestos de trabajos en la zona, vinculados principalmente a actividades domésticas. Empleadas domésticas y jardineros parecen ser los puestos más solicitados. Pero esto no es masivo. La creación de trabajos es un argumento que usualmente se emplea para defender el incremento de la diversidad social en el espacio y la gentrificación de áreas deprimidas, pero como ilustra Los Riscos, los trabajos creados por los migrantes, en su mayoría no son permanentes y tampoco bien remunerados. Como lo señala una residente:

\footnotetext{
Como te digo, a mí, por mi trabajo a mí me parece genial que llegue gente y se llene de condominios aquí. Por mi trabajo, cuanta más gente llegue acá para mi es mejor porque tengo más trabajo, a lo que me dedico. De repente hay gente de la zona que también tiene trabajo aquí mismo, para no salir para otro lado. Pero con lo que yo no estoy conforme es con mis vecinos, con ese grupo de migrantes. Encuentro que no son ningún aporte cultural (Francisca).
}

Las palabras de Francisca ilustran una posición diferente en relación al aporte cultural de los migrantes en la zona. Si bien hay acuerdo 
transversal entre los habitantes locales respecto a que el arribo de nuevos residentes ha contribuido a crear trabajos, no ocurre lo mismo con respecto al aporte cultural de estos. Algunos sostienen que los principios ecológicos que inspiran a los migrantes -especialmente a aquellos inspirados por un estilo de vida "hippie" - generan problemas con los locales. El dejar crecer libremente la vegetación es un aspecto al que recurrentemente hacen referencia con cierta molestia los locales, planteando que ello rompe con la estética del lugar, además de atraer roedores. A esto se suman las críticas respecto al papel que han tenido los migrantes con relación a la transformación de las relaciones sociales del sector. Antaño, en Los Riscos todos se conocían. Hoy, perciben en cambio que la comunidad de Los Riscos no existe. Aunque este es un fenómeno que parece estar ocurriendo en toda la sociedad, los habitantes locales sindican a los migrantes como los responsables.

Uno siente que perdió la identidad ya el sector. Uno siente como que ya no es "Los Riscos". Y como yo le contaba, antes era como una familia aquí. Por ejemplo, todas las fiestas, todas las celebraciones, todos estábamos aquí. Ahora no hay nada, todo se acabó. Yo creo que es esto mismo [la llegada de migrantes] lo que influyó para que la gente no participara (Amalia).

En la misma línea, varios entrevistados sostienen que los migrantes son individualistas, a pesar de que entre ellos perciben cierta cohesión:

No les gusta compartir con los demás (...) son individuales, son ellos, es su grupo (...) De hecho hoy como no es algo organizado por ellos [actividad de Navidad], y como ellos no entregan regalos tampoco para los niños, no ves a ninguno de ellos acá y al final disocian todo (Claudia).

Ellos no se asocian con la comunidad, pero entre ellos se conocen todos (Javier)
Las citas anteriores marcan la relación que los locales tienen con los migrantes. Dicha relación, aunque próxima en términos espaciales, es lejana en términos sociales. Esa lejanía emana desde una forma individualista de relacionarse con el medioambiente y sociedad que los rodea. Esta situación los convierte - bajo los ojos de los locales - en personas "extrañas" que practican una forma de vida que tradicionalmente no había sido observada en el sector. Ahora bien, los migrantes se conocen entre ellos. El colegio parece ser su punto de encuentro predilecto por ser un lugar donde su filosofía de vida se expresa. Pero no se relacionan mayormente con los locales. Si bien hay integración, tanto en la junta de vecinos como en el colegio y algunos puestos de trabajos, la relación entre locales y migrantes está marcada por cierta distancia, la cual en último término impide la formación de una comunidad completa cohesionada. Desde la perspectiva de los habitantes locales, lo que existe en Los Riscos es una comunidad dividida entre "ellos" (migrantes) y "nosotros" (locales).

\section{Nosotros y los migrantes: ¿Distinciones de clase $o$ autenticidad?}

Hasta aquí se ha reconocido una distinción social que opera en Los Riscos y que establece una línea simbólica que separa a los habitantes locales y los migrantes. A través de una mirada a los conflictos que hay entre ambos grupos, en esta sección se argumenta que la distinción no surge per se por las diferencias económicas que hay entre ellos, sino que más bien por un reclamo de autenticidad en torno a la pertenencia histórica al territorio.

Aunque existe una distinción entre locales y los migrantes, no se perciben mayores conflictos. 
Esto es llamativo porque si seguimos las teorías más influyentes sobre segregación, las distinciones debieran derivar en tensiones que afecten la convivencia. Pero en Los Riscos no ocurre así. Dicha condición de interacción se explica por dos factores. El primero se refiere a que tanto migrantes como locales comparten un sentido de pertenencia por el sector en el que habitan. Las condiciones medioambientales de Los Riscos y la zona que los rodea, ligan simbólicamente a ambos grupos al lugar por igual, la única diferencia entre ellos, en términos del sentido de pertenencia, radica en el tiempo en que ambos grupos han permanecido allí. Un segundo factor es la escasa interacción que hay entre migrantes y locales. Como se mostró, la sociabilidad se restringe principalmente a la Junta de Vecinos y el colegio, mientras que fuera de estos espacios la relación se restringe solo a saludos cordiales y conversaciones esporádicas. La falta de contacto puede estar dando espacio para que la diversidad de modos de vida del sector pueda estar acomodándose. Por consiguiente, tanto el sentido de pertenencia a un territorio común como la falta de contacto, son aspectos que crean una base de entendimiento que suaviza los potenciales conflictos que puedan derivar de la distinción entre locales y migrantes. Consultadas sobre cómo es la relación con los nuevos residentes, entrevistadas sostienen:

\footnotetext{
Mala relación nosotros no tenemos, por lo menos con la gente que ha tenido más contacto con el sector. La junta de vecinos es la que trabaja, en ese momento se encuentra la gente. Nosotros no tenemos mayor problema con la convivencia. No tenemos mala relación. Yo tendría que mentirle si le digo que nos llevamos mal (Verónica).

Investigador: ¿Cuándo se juntan con ellos?

En las reuniones de las juntas de vecinos. Pero te diré que los únicos que llegan son los de la directiva, porque los otros no vienen acá. Si uno se los encuentra en la calle te saludan, pero nada más (Amalia).
}

Duranteel trabajo en terreno no se logróacreditar la existencia de conflictos de clase. Como ha sostenido la literatura sobre segregación, en entornos socioeconómicamente diversos tienden a emerger relaciones marcadas por el clasismo que derivan en discriminación desde arriba hacia abajo. Este argumento, que suele ser empleado contra la diversidad social del espacio, no se sostiene para el caso de estudio. Contrario a lo esperado, la mayor parte de los migrantes fueron reconocidos por los locales como individuos que favorecen la integración. Dicha actitud fue relacionada con la concepción del mundo que ellos tienen, aspecto que, en opinión de los entrevistados, le permite desprenderse de los prejuicios asociados a las condiciones de clase y construir relaciones sociales más allá de los bienes materiales que poseen las personas. Así, el individualismo al que hizo referencia en el testimonio anterior y que parece marcar el ritmo de las relaciones que los migrantes mantienen con los habitantes locales, está acompañado también de una actitud en la cual la situación socioeconómica de las personas pierde relevancia como categoría estructuradora de las relaciones sociales.

Hasta aquí la discusión apunta a una situación ideal. La distinción o segregación cultural no está motivada por razones de clase y no interviene destructivamente en la interacción entre migrantes y locales. Pero cabe preguntar si la distinción entre ellos y nosotros -enunciada previamente- solo puede ser entendida a partir de diferencias en los estilos de vida o bien existe algo más. Para ello, se preguntó también respecto a cómo se manifestaba la relación con los migrantes en un espacio de encuentro tradicionalmente local: la Junta de Vecinos. Desde aquí emanó el principal conflicto que 
enfrenta a ambos grupos y los aspectos desde los cuales se entiende la distinción comentada.

Según relatan los entrevistados, en los últimos años la Junta de Vecinos se ha convertido en un espacio de disputa política entre migrantes y locales. En este espacio, que los entrevistados reconocen como propio, los migrantes han intentado liderar proyectos que divulguen sus principios e ideas en la zona. Sin embargo, esta situación es muchas veces resistida por la comunidad local:

\begin{abstract}
Toman bandera de cosas no tan relevantes. Por decirte, nosotros preferimos arreglar el camino. Ellos se inclinan por un proyecto ecológico de la basura. Entonces, todos tienen distintas visiones de las cosas. Ellos deben entender una cosa, que tú no puedes llegar y cambiarle la gente la forma de pensar. Sí aconsejarlos y tratar de que te entiendan, pero no puedes llegar a una comunidad que son antiguos todos, que llevan aquí años, que son hijos de los hijos y que llevan aquí mucho tiempo. Tú no puedes llegar así con tu forma tan radical y querer cambiar eso. Puedes intentarlo, pero de a poco. No es llegar e instalar tus ideas.
\end{abstract}

Investigador: ¿se sienten desplazados?

Si. Ellos intentan imponer su estilo en vida en la zona (Javier).

La crítica que los locales realizan a los migrantes no está dirigida al estilo de vida que ellos tienen, sino que más bien a que están ocupando un espacio "perteneciente" a los locales para divulgar sus ideas. La junta de vecinos, como espacio de poder, ha comenzado a ser controlada por los migrantes, por eso el sentimiento de desplazamiento que manifiestan.

La junta de vecinos es sólo un espacio donde se manifiesta la distinción entre locales y migrante. El cuerpo de esta distinción está en las prácticas cotidianas marcadamente diferentes que se producen entre ambos grupos. Esto último se manifiesta de muchas formas. Discrepancias en torno a dejar crecer libremente la vegetación, diferencias en torno al horario de las fiestas comunitarias, discrepancias en torno al faenamiento de animales, entre otras. En el contexto de estas discrepancias y producto de la migración masiva, los locales han pasado a ser minoría. Por lo tanto, algunas actividades que hace 10 o 15 años atrás eran normales para los habitantes locales, hoy simbólicamente se han convertido en actividades problemáticas para los habitantes del sector. Este desplazamiento simbólico respecto a la forma de vida que tienen los locales ha causado malestar entre estos, alimentando así la distinción con los migrantes. Ese desplazamiento y malestar se observa en las palabras de los entrevistados:

Lo peor que tiene ellos es que vienen de afuera y con otras ideas, que quieren ellos venir a cambiar a la gente (Camila).

Ellos no quieren adecuarse al sector donde llegaron, sino que cambiar al resto que llevamos toda una vida aquí (Amalia).

En estos testimonios subyace un reclamo de autenticidad por parte de los habitantes locales. Por su actual condición de minoría, estos han comenzado a ser desplazados desde las posiciones de poder local y algunas de sus prácticas han sido cargadas de un sentido social negativo. En ese contexto, emerge el reclamo de los habitantes locales hacia los migrantes. Este reclamo está permeado por el tiempo de permanencia de cada grupo en el lugar. En el imaginario de los entrevistados el tiempo entrega posesión, otorgándole un sentido de propiedad al lugar. Ellos dicen ser los auténticos, pertenecer a este lugar y no así los migrantes, quienes son vistos como afuerinos o recién llegados. 
La distinción entre "ellos" y "nosotros" que manifiestan los entrevistados, no emerge así desde las diferencias económicas per se, sino más bien desde la relación misma que se ha forjado entre locales y migrantes. Como sugieren los resultados, esa diferenciación que hacen los locales está enraizada en un sentido de desplazamiento simbólico de las posiciones de poder y el consiguiente reclamo de autenticidad que despliegan como reacción a ese proceso.

\section{Conclusión. Migración por estilo de vida y comunidades segregadas: espacios abiertos al cambio.}

En este artículo se examinó cómo opera la segregación sociocultural en una pequeña localidad suburbana que ha sido objeto de migración por estilo de vida durante el último decenio. Se ha comprobado que paralelo al arribo de nuevos vecinos, se ha desarrollado un proceso de desplazamiento de la población que históricamente ha habitado en el sector y un incremento en la mixtura social del mismo, dado el arribo de sujetos preferentemente de clase media y alta. Estos resultados sugieren que el lugar está experimentando un proceso de gentrificación, pero que a diferencia de lo que ocurre en los espacios urbanos, ha estado motivado por migrantes que arrastran un discurso ecologista activo. Dicho grupo es reconocido, primero, como una comunidad homogénea en términos del estilo de vida, y segundo, como extraña, por cuanto sus prácticas cotidianas se alejan de las que históricamente han caracterizado a los habitantes locales. Este panorama configura una distinción social entre lugareños y migrantes que imposibilita la construcción de una comunidad diversa y cohesionada y que se refuerza a partir de reclamos de autenticidad sobre el territorio en el que habitan ambos grupos.

Al igual que la literatura existente, los resultados indican que la migración por estilo de vida puede inducir procesos de segregación sociocultural en los lugares de acogida. Sin embargo, los resultados también muestran que dicha segregación no se explica per se por la diversidad socioeconómica que induce este tipo de migración, sino que más bien emerge a partir de la relación cotidiana entre los grupos. Este resultado lleva a situar el origen y mantenimiento de la segregación a nivel microsocial y no en estructuras determinadas por la clase social. Como Bauman (2002) ha argumentado, la clase social hoy ha perdido relevancia para explicar las identidades colectivas y en ese contexto, como ilustra este artículo, el estilo de vida y los reclamos de autenticidad toman fuerza para entender el surgimiento de distinciones sociales.

Ahora bien, esto no significa que la clase social esté completamente ausente de las relaciones sociales, ya que puede estructurar los estilos de vida (Bourdieu 2002) y valores individuales (Inglehart 1990). Lo que los resultados sugieren es que a pesar de que la clase influye en la manera en que los sujetos se relacionan con otros, esta no es una categoría que determine por si sola las relaciones sociales. La manera en que se produce el contacto entre residentes en la cotidianeidad, parece ser un elemento más importante que la clase en la cohesión social de una comunidad socioeconómicamente diversa.

Un resultado llamativo en esta investigación -y que sustenta futuras investigaciones - es que, a pesar de la existencia de segregación sociocultural y formas de distinción social, no se verifican conflictos activos en el lugar. 
Este resultado reafirma el argumento de que la diversidad socioeconómica no tiene que inducir necesariamente conflictos a partir de la estructuración de clase. Así, dado que la segregación es un proceso anclado a nivel microsocial, este no tendría que ser necesariamente un fenómeno inevitable. De hecho, como ilustra el caso de estudio, la comunidad -más allá de sus diferencias socioeconómicas- se podría articular de forma conjunta sobre la base de una misma valoración del paisaje y la naturaleza, así como también sobre la defensa de lo prístino de un lugar crecientemente acosado por la mercantilización y el negocio inmobiliario. Se podrían desarrollar así "alianzas improbables" (Grossman 2005) que terminen cohesionando la comunidad más allá de sus diferencias. Aquí emerge un espacio de intervención política interesante que es necesario aprovechar con el fin de reducir la segregación en lugares que avanzan en diversidad producto del arribo de nuevos vecinos, sean en entornos urbanos o rurales. Sin embargo, sin espacios de encuentro donde migrantes y habitantes locales se puedan reconocer como pares, en posiciones similares y sin disputas de poder, se limita la posibilidad de construir cohesión social.

La noción "reclamo de autenticidad" es útil para entender cómo opera la segregación sociocultural en lugares receptores de migración por estilo de vida. Así como los migrantes suelen reclamar naturaleza, conservación y respeto ambiental (Benson 2013), el reclamo de los lugareños se sustenta en la ocupación histórica del lugar. Dichos reclamos -que pueden tomar las más diversas formas- definen el núcleo y los límites de las aspiraciones y prácticas cotidianas de quienes conviven en un mismo lugar $y$ que, como ocurre en Los Riscos, se separan simbólicamente. La existencia de diferentes reclamos de autenticidad no es un fenómeno exclusivo a los lugares receptores de migración por estilo de vida, sino que también puede ser encontrado en barrios urbanos gentrificados, en donde conviven sujetos portadores de diferentes racionalidades, intereses y estilos de vida. Esto último es importante en términos de la investigación futura, especialmente en Chile y Latinoamérica donde estos fenómenos están poco estudiados aún. A pesar de que autores han criticado el uso del concepto gentrificación para explicar los cambios en zonas rurales, son varias las similitudes que tiene este fenómeno con aquello que ocurre en espacios urbanos. Se necesita más reflexión e investigación orientada específicamente hacia el reconocimiento de las diferentes "geografías de la gentrificación" (Phillips 2004), como también avanzar en una conceptualización de este fenómeno adecuada para el hemisferio sur (López-Morales 2018). 


\section{Bibliografía}

Ariztía, T. 2009. "Arreglando la casa. La cultura de la movilidad en Chile". En Pérez, F. \& Tironi, M. Espacios, prácticas y cultura urbana (pp. 222-240). Santiago: Arq.

Bauman, Z. 2002. Modernidad líquida. México D.F: Fondo de Cultura Económica.

Bayón, M. y Saraví, G. 2013. "The cultural dimensions of urban fragmentation: Segregation, sociability, and inequality in Mexico City". Latin American Perspectives, 40(2), 35-52

Bell, D., y Jayne, M. 2010. "The creative countryside: Policy and practice in the UK rural cultural economy". Journal of rural studies, 26(3), 209-218.

Bennett, D. 2010. "Creative migration: A Western Australian case study of creative artists". Australian Geographer, 41(1), 117128.

Benson, M. 2013. "Living the "Real" Dream in la France profonde?: Lifestyle Migration, Social Distinction, and the Authenticities of Everyday Life". Anthropological Quarterly, 86(2), 501-525.

Benson, M. y O'reilly, K. 2009. "Migration and the search for a better way of life: a critical exploration of lifestyle migration". The sociological review, 57(4), 608-625.

Bernt, M. 2018. "Gentrification between urban and rural". Dialogues in Human Geography, 8(1), 31-35

Bourdieu, P. 2002. La distinción. Criterio y bases sociales del gusto. México D.F: Taurus.

Cassiers, T. y Kesteloot, C. 2012. "Socio-spatial inequalities and social cohesion in European cities". Urban Studies, 49(9), 1909-1924.

Espinoza, V. y Barozet, E. 2009. “¿De qué hablamos cuando decimos "clase media"? Perspectivas sobre el caso chileno". En Joignant, A., y Güell, P. El arte de clasificar a los chilenos. Enfoques sobre los modelos de clasificación en Chile (pp. 103130). Santiago: Ediciones Universidad Diego Portales

Florida, R. 2005. Cities and the creative class. New York: Routledge.

Goffman, E. 1974. Frame analysis: An essay on organization of experience. Cambridge: Harvard University Press.

González, R. 2011. "Los procesos de migración de amenidad y la competitividad de destinos turísticos de montaña del oeste canadiense y de la Norpatagonia argentina". Estudios y perspectivas en turismo, 20(5), 1102-1122.

González, R., Otero, A., Nakayama, L. y Marioni, S. 2009. "Las movilidades del turismo y las migraciones de amenidad: problemáticas y contradicciones en el desarrollo de centros turísticos de montaña". Revista de Geografía Norte Grande, (44), 75-92.

Grossman, Z. 2005. "Unlikely alliances: treaty conflicts and environmental cooperation between Native American and rural white communities". American Indian culture and research journal, 29(4), 21-43.

Halfacree, K. 2018. "From Ambridge to the world? Class returns to rural population geographies". Dialogues in Human Geography, 8 (1), 26-30.

Hayes, M. 2015. "Into the universe of the hacienda: lifestyle migration, individualism and social dislocation in Vilcabamba, Ecuador". Journal of Latin American Geography, 14 (1), 79-100.

Hidalgo, R. y Zunino, H. 2011. "Negocio inmobiliario y migración por estilos de vida en la Araucanía lacustre: la transformación del espacio habitado en Villarrica y Pucón”. AUS, (11), 10-13.

Inglehart, R. 1990. Culture Shift. New Jersey: Princeton University Press.

Inzulza-Contardo, J. 2012. 'Latino gentrification'?: Focusing on physical and socioeconomic patterns of change in Latin American inner cities". Urban Studies, 49(10), 2085-2107

Janoschka, M. 2009. "The contested spaces of lifestyle mobilities: regime analysis as a tool to study political claims in Latin American retirement destinations". Die Erde, 140(3), 251

Janoschka, M., Sequera, J. y Salinas, L. 2014. "Gentrification in Spain and Latin America-A critical dialogue". International Journal of Urban and Regional Research, 38(4), 1234-1265.

Kordel, S. y Pohle, P. 2018. "International Lifestyle Migration in the Andes of Ecuador: How Migrants from the USA Perform Privilege, Import Rurality and Evaluate Their Impact on Local Community". Sociologia Ruralis, 58(1), 126-146.

Korpela, M. 2014. "Lifestyle of freedom? Individualism and lifestyle migration". En Benson, M., y Osbaldiston, N. Understanding lifestyle migration (27-46). London: Palgrave Macmillan.

López-Morales, E. 2013. "Gentrificación en Chile: aportes conceptuales y evidencias para una discusión necesaria". Revista de Geografía Norte Grande, (56), 31-52.

López-Morales, E. 2018. "A rural gentrification theory debate for the Global South?". Dialogues in Human Geography, 8(1), 47-50.

Manzi, T. y Smith-Bowers, B. 2005. "Gated communities as club goods: segregation or social cohesion?". Housing Studies, 20(2), 345-359.

Marchant, C. 2017. "Lifestyle Migration and the Nascent Agroecological Movement in the Andean Araucanía, Chile: Is It Promoting Sustainable Local Development?". Mountain Research and Development, 37(4), 406-414

Marchant, C. y Rojas, F. 2015. "Transformaciones locales y nuevas funcionalidades económicas vinculadas a las migraciones por amenidad en la Patagonia chilena. El caso de Malalcahuello, una villa de montaña". Journal of Alpine Research, 103(3).

Massey, D. y Denton, N. 1988. "The dimensions of residential segregation”. Social forces, 67(2), 281-315.

Matarrita-Cascante, D., y Stocks, G. 2013. "Amenity migration to the global south: Implications for community development". Geoforum, 49, 91-102.

Matarrita-Cascante, D., Zunino, H., y Sagner-Tapia, J. 2017. "Amenity/Lifestyle Migration in the Chilean Andes: Understanding 
the Views of "The Other" and Its Effects on Integrated Community Development". Sustainability, 9(9), 16-19.

Matossian, B., Zebryte, L., y Zunino, H. 2014. "Europeos y estadounidenses en la Norpatagonia andina: nuevas experiencias". Revista CIDOB d'afers internacionals, 237-259.

Méndez, M. 2014. "Una tipología de los nuevos habitantes del campo: aportes para el estudio del fenómeno neorrural a partir del caso de Manizales, Colombia". Revista de Economia e Sociologia Rural, 51, 31-48.

Monkkonen, P. 2012. "La segregación residencial en el México urbano: niveles y patrones". Eure, 38(114), 125-146.

Nates, B. 2008. "Procesos de gentrificación en lugares rururbanos: presupuestos conceptuales para su estudio en Colombia". Antropol.social, (10), 253-269.

Nogué, J. 1988. "El fenómeno neorural". Agricultura y Sociedad, 47, 145-175.

Okuda, M. y Gómez-Restrepo, C. 2005. "Métodos en investigación cualitativa: triangulación". Revista colombiana de Psiquiatría, 34(1), 118-124

Otero, A. Zunino, H. y Rodríguez, M. 2017. "Las tecnologías socioculturales en los procesos de innovación de los migrantes de amenidad y por estilos de vida: El caso del destino turístico de Pucón, Chile". Revista de Geografía Norte Grande, (67), 211-233.

Phillips, M. 2004. "Othergeographies of gentrification". Progress in human geography, 28(1), 5-30.

Phillips, M. 2005. "Differential productions of rural gentrification: illustrations from North and South Norfolk". Geoforum, 36(4), 477494.

Rasse, A. 2015. "Juntos pero no revueltos: procesos de integración social en fronteras residenciales entre hogares de distinto nivel socioeconómico". Eure, 41(122), 125-143.

Ruiz-Tagle, J. 2016. "La persistencia de la segregación y la desigualdad en barrios socialmente diversos: un estudio de caso en La Florida, Santiago". Eure, 42(125), 81-108.

Ruiz-Tagle, J., Labbé, G., Rocco, V., Schuster, J. y Muñoz, J. 2018. Recuperación de plusvalía para financiar la inserción de viviendas sociales en barrios consolidados. Centro de Políticas Públicas UC.

Sabatini, F., Cáceres, G., y Cerda, J. 2001. "Segregación residencial en las principales ciudades chilenas: Tendencias de las tres últimas décadas y posibles cursos de acción”. Eure, 27(82),
21-42.

Sabatini, F., Robles, M. S., y Vásquez, H. 2009. "Gentrificación sin expulsión, o la ciudad latinoamericana en una encrucijada histórica". Revista 180, (24).

Sánchez, R. 2017. "Leisure-tourism and amenity migration: transformations observed in the semiarid mountain region of Northern Chile". Journal of Tourism and Cultural Change, 1-16

Saraví, G. A. 2008. "Mundos aislados: segregación urbana y desigualdad en la ciudad de México". Eure, 34(103), 93-110.

Stockdale, A. 2010. "The diverse geographies of rural gentrification in Scotland". Journal of Rural Studies, 26(1), 31-40.

Trimano, L. 2017. "Paisajes y gringos: Neorruralidad serrana, transformaciones relacionales e identidades emergentes". Chungará, 49(3), 461-471.

Urrutia-Mosquera, J., López-Ospina, H., Sabatini, F. y Rasse, A. 2017. "Tolerancia a la diversidad y segregación residencial. Una adaptación del modelo de segregación de Schelling con tres grupos sociales". Eure, 43(130), 5-24.

Vergara-Erices, L., y Garín, A. 2016. "Vivienda social y segregación socioespacial en una ciudad pequeña: el caso de Angol, Chile". Polis, 15 (44).457-486.

Vergara, L., Rozas, M. y Zunino, H. 2013. "Los imaginarios urbanos y la arquitectura de Puerto Varas: encrucijada entre lo local y lo global". AUS, (14), 19-22.

Vignoli, J., y Rowe, F. 2017. “¿Contribuye la migración interna a reducir la segregación residencial?: el caso de Santiago de Chile 1977-2002". Revista Latinoamericana de Población, 11(21), 7-46.

Villarreal, A. y Silva, B. 2006. "Social cohesion, criminal victimization and perceived risk of crime in Brazilian neighborhoods". Social Forces, 84(3), 1725-1753.

White, M. 1983. "The measurement of spatial segregation". American journal of sociology, 88(5), 1008-1018.

Zunino, H., Hidalgo, R., y Marquardt, E. 2011. "Vivienda social y segregación espacial en la Ciudad de Pucón, Chile: entre el enclaustramiento y la integración con el hábitat turístico". Revista INVI, 26(71), 15-55.

Zunino, H. y Huiliñir, V. 2017. "Utopías Modernas y posmodernas en el Sur de Chile: rupturas y continuidades". En: Sánchez, R., Hidalgo, R. y Arenas, F. (Eds.) Re-conociendo las geografías de América Latina y el Caribe (pp. 157-182). Santiago, Pontificia Universidad Católica de Chile. 
